\title{
Defective apoptosis and tumorigenesis: role of p53 mutation and Fas/FasL system dysregulation
}

\author{
F. Cappello, M. Bellafiore, A. Palma, and F. Bucchieri
}

Sezione di Anatomia Umana, Dipartimento di Medicina Sperimentale, Università di Palermo, Italy

Accepted: 14/03/02

Key words: apoptosis, p53, Fas, FasL, tumorigenesis, gene therapy

\section{SUMMARY}

The transcription factor p53 and the cytokine receptor FasL are two of the most famous regulators of cell life, and their alterations can cause a large number of pathologies, including cancer. In this review, we focused on how they can determine defective apoptosis, one of the causes of tumorigenesis and tumor progression. The importance of this knowledge lies in the new perspectives that gene therapy can offer to cure cancer.

\section{INTRODUCTION}

The term "apoptosis" was first used by Kerr et al. (1972) to describe a distinctive and important type of cell death, also called programmed cell death. The word "apoptosis" derives from Greek and describes, literally and poetically, the ultrastructural changes observed in a cell during this phenomenon: the cell looks like a flower dropping its petals. Indeed, nuclear condensation and fragmentation followed by the cytoskeletal disruption and membrane blebbing give this feature to apoptotic cells both in vivo and in vitro (Fig. 1). Nevertheless, this phenomenon was probably first observed by Flemming (1885) in normal maturating rabbit ovarian follicles; he described the crescents of chromatin, a typical feature of apoptotic cells and called this phenomenon chromatolysis. The historical development of the cell death concept was previously elegantly illustrated by Majno and Joris (1995) and we refer to it for details.

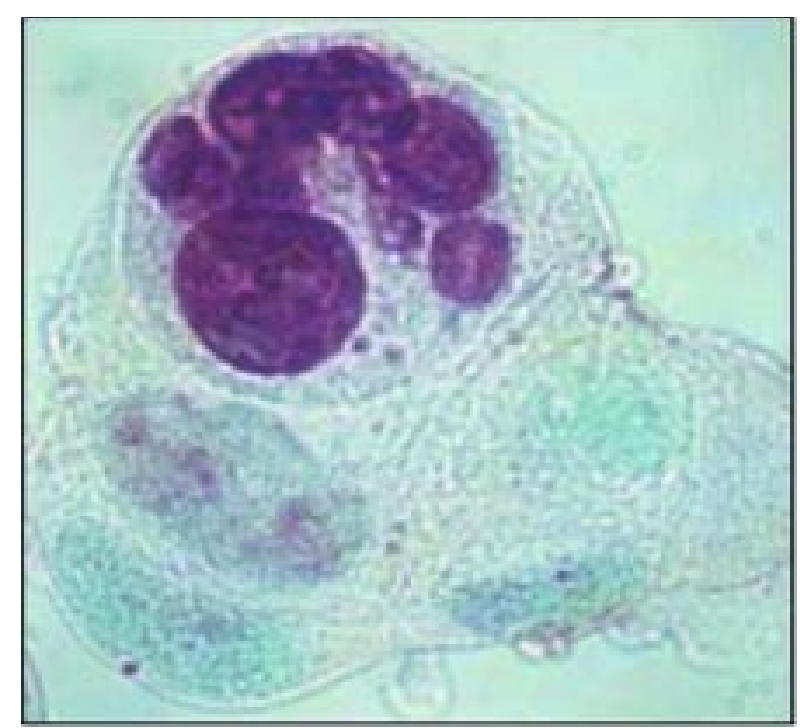

Fig. 1 - The cell in the upper portion is apoptotic as demonstrated by the positivity of its nucleus for the TUNEL (TdT UTP Nick End Labelling) staining together with the typical morphological features (nuclear condensation and fragmentation). The other four cells are viable. 100X 
Apoptosis is a physiologic event that occurs normally during embryogenesis, organogenesis and maintenance of tissue homeostasis, as well as in ageing (Saunders and Fallon, 1967; Server and Mobley, 1991; Budtz, 1994). Nevertheless, various pathologies derived from a deregulated triggered apoptosis, i.e. AIDS (Pantaleo et al., 1993), Alzheimer's disease (Mattson et al., 1992), cardiovascular diseases (Condorelli et al., 1999) and some genetic disorders, such as Farber disease (Farina et al., 2000), share the presence of uncontrolled apoptotic phenomena, while apoptosis is faulty in some autoimmune disorders (Watanabe-Fukunaga et al., 1992; Cohen et al., 1992, Rovere et al., 2000) and cancer.

The possible implication of apoptosis in neoplastic growth was first suggested by Danilevicius (1973), and in the last thirty years many researchers have focused on the possible mechanisms determining block of pro-apoptotic signals in cancerous cells.

Apoptosis can be triggered a) extrinsically, by DNA or mitochondrial damage, such as following radiation, toxin or free radical exposure, or b) intrinsically, via membrane receptor-ligand interactions (Fig. 2). Both mechanisms activate caspases, a fam-

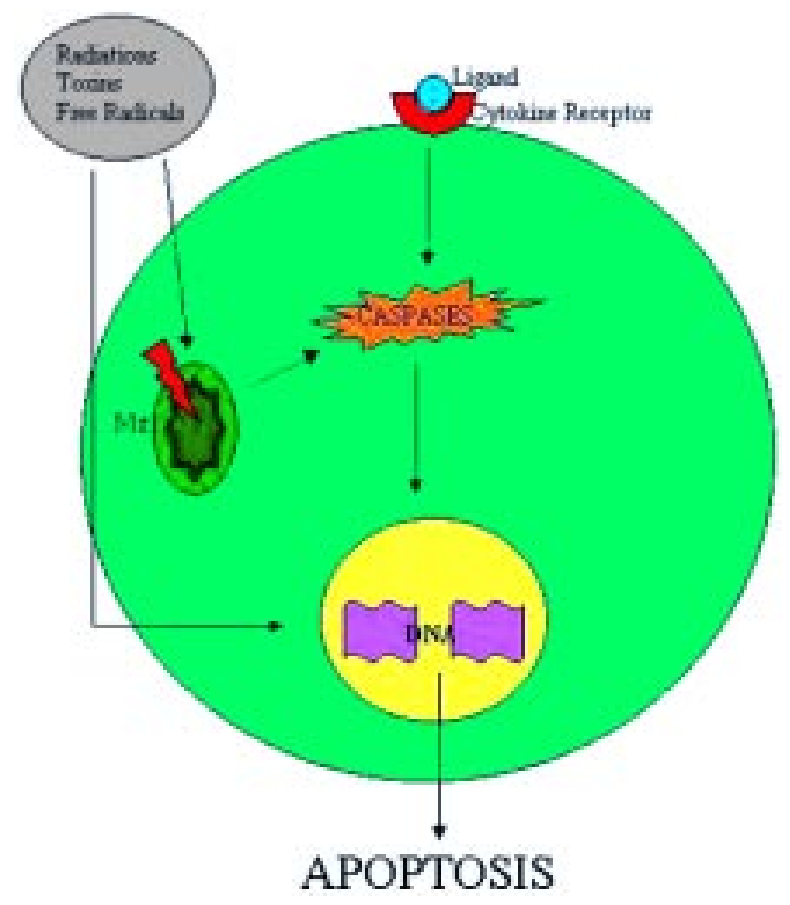

Fig. 2 - Radiations, toxics and/or free radicals can trigger apoptosis by DNA and/or mitochondrial (Mit) damage. The latter induces activation of caspases, as well as some membrane receptor-ligand interactions which execute apoptosis. ily of cysteine aspartic proteases, functionally divided in two groups (initiators and executors), that induce proteolysis of cellular proteins and determine apoptotic cell death (Alnemri et al., 1996; Martin and Green, 1995; Kumar and Lavin 1996).

In this review, we describe p53 mutation and Fas/FasL system deregulation as models of defective apoptosis during carcinogenesis and tumor progression. We focus our attention particularly on the prognostic significance of these molecular alterations, highlighting the new therapeutic tools that a better knowledge of these mechanisms could offer.

\section{DEFECTIVE APOPTOSIS AS A CONSE- QUENCE OF P53 MUTATION}

Many apoptotic stimuli are signalled to cells through transcription factors (i.e. AP1, IRF-1 and NF-kB) and mediated by regulatory molecules, such as p53 and rb. The transduction of these stimuli leads to the activation of the caspases and induction of apoptosis. p53 the "guardian of the genome", is a tumor suppressor gene that produces a nuclear phosphoprotein, whose half-life is normally very short. When a mutagenic agent hits a cell, p53 blocks the cell cycle, allowing DNA repair. However, if the repair process fails, p53 induces the expression of pro-apoptotic genes, among which is bax (Table 1). Nevertheless, when p53 is mutated, it loses its function, its half-life becomes longer, and it accumulates at the nuclear level; the DNA-damaged cells can therefore continue to proliferate, resulting in cancer development (Pellegata and Ranzani, 1996) (Fig. 3).

p53 over-expression has been observed in many malignant tumors, such as thyroid tumors (Hermann et al., 2001), Ewing sarcoma (de Alava et al., 2000), paediatric rhabdomyosarcoma (Taylor et al., 2000), non-small cell lung cancer (Lavezzi et al., 1999), invasive breast carcinoma (van Slooten et al., 1999), astrocytoma (Watanabe et al., 1997) and tongue cancer (Atula et al., 1996). Generally, p53 alterations appear to be associated with a markedly poor outcome. Nevertheless, over-expression of wild-type p53 may be correlated with a deregulation without loss of function (Hermann et al., 2001). In turn, this could be the reason for the good prognosis of some patients with cancer, even 
Table I

Pro-apoptotic gene substrates of p53

\begin{tabular}{lll}
\hline Gene & \multicolumn{1}{c}{ Signaling pathway } & Effect \\
\hline Bax & Release of mitochondrial cytochrome C & Apoptosis \\
$I G F-B P 3$ & Inhibit growth factor signals, binding to IGF1 & Apoptosis \\
FAS/APO1 & Use FADD as to activate caspase cascade & Apoptosis \\
KILLER/DR5 & Caspase cascade & Apoptosis \\
$P I G 1-P I G 14$ & Oxidative stress & Apoptosis \\
p85 & Oxidative stress & Apoptosis \\
PAG608 & $? ? ?$ & Apoptosis \\
\hline
\end{tabular}

though wild-type p53 over-expression is not always related with a favourable outcome (Taylor et al., 2000; Ryan et al., 2001).

The prognostic significance of p53 mutation has been observed in many tumors, (including gastric cancer (Lam, 1999; Matturri et al., 1998), astrocytoma (Nokazi et al., 1999), non-small cell lung cancer (Bennett et al., 1998), breast carcinoma (Powell et al., 2000; Hurlimann et al., 1995), ovarian cancer (Laframboise et al., 2000). These studies have found a reduction of apoptotic phenomena associated to p53 mutation and poor prognosis. p53 abnormality had the potential for screening, early detection and prognostication (Lam, 1999). Indeed, p53 may be useful in predicting the malignancy of each of these tumors. Furthermore, it helps in identifying patients that need additional peri-operative therapies (Matturri et al., 1998). Moreover, a more advanced surgical stage or tumor grade were suggestive of higher rates of p53 mutations (Laframboise et al., 2000), determining a more aggressive surgery.

Genetic diagnosis of p53 mutation can also permit the evaluation and design of specific therapies to prevent malignant progression (Nokazi et al., 1999). Indeed, such data have provided clinical support for treatment with anticancer drugs

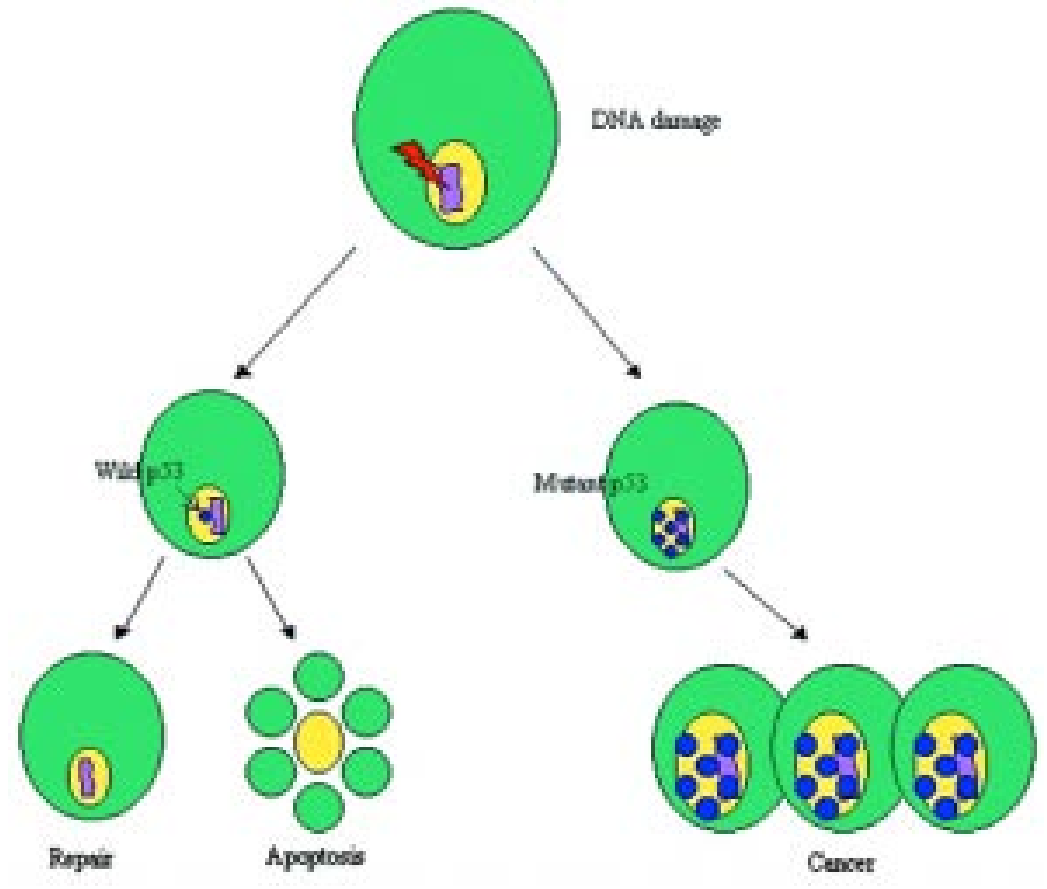

Fig. 3 - When DNA is damaged, wild-type p53 can determine DNA repair. If this process falls, wild-type p53 induces apoptosis. Mutant p53 can not induce either cellular repair or apoptosis, determining proliferation of neoplastic clones. 
(Chang and Lai, 2001; King et al., 2000), hormonal therapy (Hurlimann et al., 1995) or radiation (Farid, 2001), because of the role that p53 has in predicting one's response to therapy (Velculescu and El-Deiry, 1996). In addition, the use of cisplatin and doxorubicin in bladder cancer (Chang and Lai, 2001), as well as paclitaxel in metastatic non-small cell lung carcinoma (King et al., 2000) was suggested by the observation that these drugs can activate apoptosis in the absence of wild-type $\mathrm{p} 53$, probably inducing cell death by an alternative pathway.

We have compared p53 expression in 8 soft tissue tumors with different prognoses, 4 biphasic synovial sarcomas of different sites and 4 pleural biphasic malignant mesotheliomas (Cappello and Barnes, 2001), finding that p53 was expressed more strongly in the tumors with the poorer prognosis (synovial sarcomas) (Fig. 4). We confirmed the over-expression of p53 also in another variant of synovial sarcoma, the poorly differentiated type (Cappello et al.,

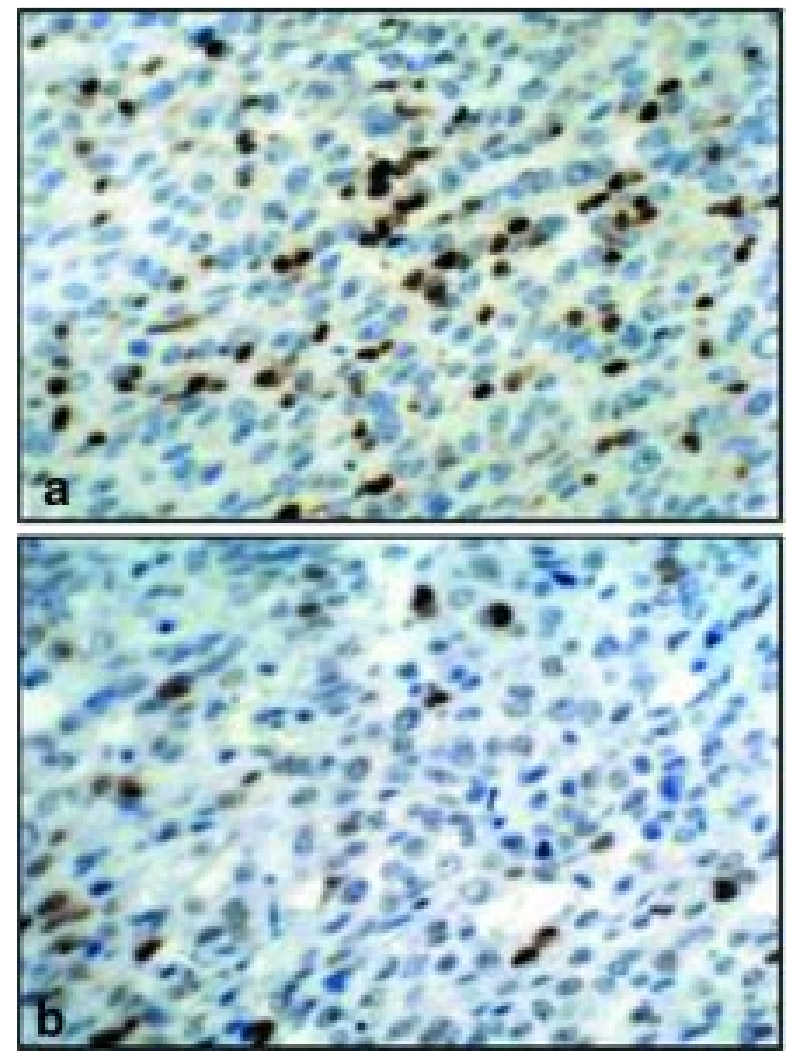

Fig. 4 - p53 expression can have a prognostic significance: synovial sarcomas (a) have a worse prognosis than malignant mesotheliomas (b). M.O. 40X, chromogen: DAB.
2001). In all observed synovial sarcomas, apoptotic phenomena were inconspicuous, suggesting a role of defective apoptosis in pathogenesis and progression of these tumors. Moreover, in another study (Cappello et al., 2002), we compared p53 expression in 63 prostate tumors (Gleason 6) exhibiting both tubular and cribrous patterns. We found an identical expression of p53 in both patterns, confirming the validity of the Gleason grading system to include them in the same grade and to approximate the morphologic grade with biologic potential and prognostic value. Moreover, we found a linear relationship between an increase in PCNA (proliferating index) positivity and p53 expression in these 63 prostatic cancers, suggesting that p53 mutation was a late event in prostate cancerogenesis and could be related to the unfavourable prognosis of the prostatic cancer in an advanced stage. In all studies, we used monoclonal antibodies that recognised both wild-type and mutant p53; however, since the wild type protein is rapidly degraded, its physiologic levels remain below the detectable immunohistochemical threshold. For this reason, p53 immunoreactivity reflected p53 gene mutations.

It is also necessary to consider that the transduction of p53-mediated pro-apoptotic stimuli is regulated by many proteins, among which is bcl-2. Bcl-2 is normally expressed in mitochondria, endoplasmic reticulum and nuclear envelope. Mitochondrial bcl2 forms homodimers that bind a pro-apoptotic protease-activating factor (APAF-1), which in turn is bound to caspase-9. Bcl-2 suppresses apoptosis both by preventing the increase in mitochondrial membrane permeability (direct way) and by interacting with other pro-apoptotic proteins (mediated way). When DNA is irreversibly damaged, p53 induces the synthesis of bax, which binds bcl-2, forming a bax-bcl-2 heterodimer. The result is an increase in permeability of mitochondrial membranes and the release of the cytocrome $\mathrm{C}$ which disrupts the bcl2/APAF-1 complex and primes the caspase-cascade, triggering apoptosis (Fig. 5). An over-expression of bcl-2 can inhibit apoptotic phenomena in vitro (Bissonnette and Echeverri, 1992). Moreover, an inverse correlation between bcl-2 expression and apoptotic events has been confirmed in vivo (Tormanen et al., 1995; Kulig et al., 1999). In our study, bcl-2 was more positive in synovial sarcoma, while bax resulted more expressed in malignant mesothelioma, confirming that a defective apoptosis in synovial sarcoma could explain the worse prognosis, compared to 
IMM OMM

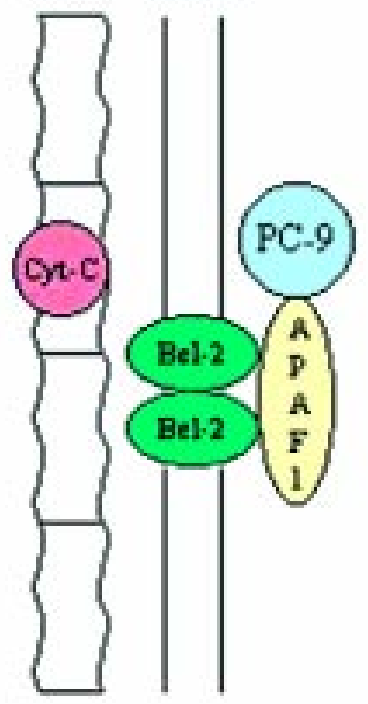

Nuclear damage



\section{IMM OMM}

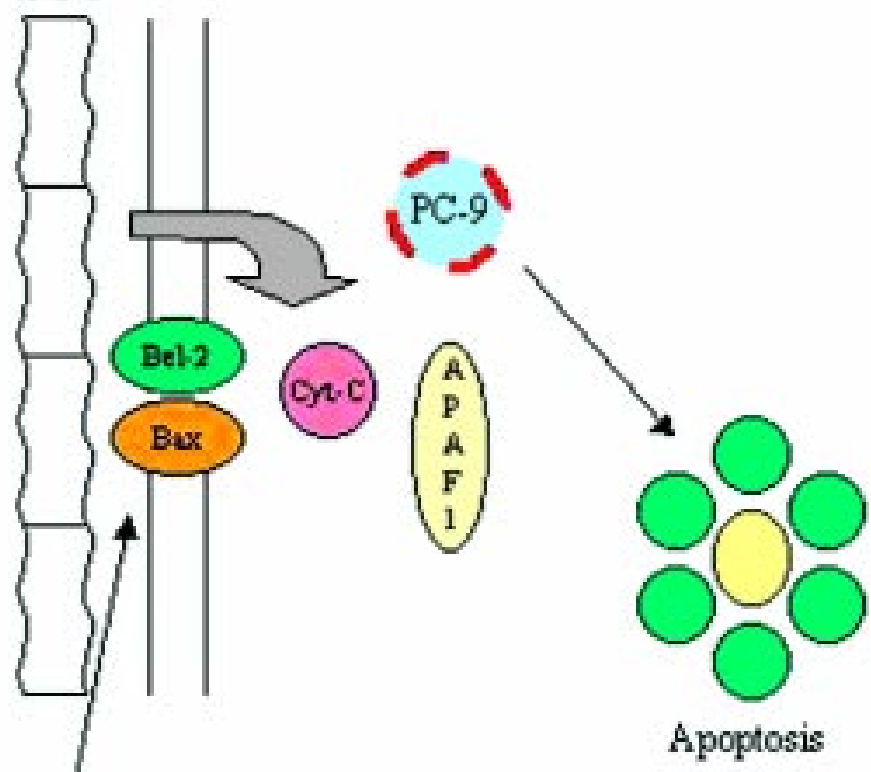

Fig. 5 - Bcl-2 homodimer binds APAF-1, which in turn is bound to pro-caspase 9 (PC-9) on outer mitochondrial membrane (OMM) This complex stabilizes mitochondrial membrane permeability. When DNA is irreversibly damaged, wild-type p53 induces synthesis of bax that binds bcl-2 forming a heterodimer. This determines the increase of inner mitochondrial membrane (IMM) permeability and the release of cytochrome $\mathrm{C}(\mathrm{Cyt}-\mathrm{C})$ that disrupts bcl-2- APAF-1 complex and activates pro-caspase 9, initiating apoptosis.

malignant mesothelioma. These data suggested also the importance of associating several pro- and antiapoptotic markers to p53 expression to better elucidate the apoptotic status of observed tumors.

\section{DEFECTIVE APOPTOSIS AS A CONSE- QUENCE OF FAS/FASL DYSREGULATION}

Apoptotic stimuli are signalled to the nucleus also through membrane receptors such as Fas and TNF. In particular, Fas, also called APO-1 or CD95, is a cell surface protein belonging to the TNF/NGF receptor family (Itoh et al., 1991; Oehm et al., 1992, Nagata, 1993). Fas is constitutively expressed in many tissues, such as thymus, liver, heart and lung (Watanabe et al., 1991), where it is involved in homeostasis. An abnormal activation of Fas in these tissues can cause severe damage, such as fulminant hepatitis (Ando et al., 1993). The involvement of Fas and its ligand (FasL) has been proven in many human autoimmune diseases, where it has also been demonstrated that FasL (Fas ligand) is the death factor and Fas is its receptor (Nagata, 1994). Indeed, the apoptotic signal is induced by the binding of FasL to Fas. Death signalling requires clustering of Fas with a cytoplasmic adapter protein which exhibits a corresponding death domain (FADD, or Fas-associated death domain). In turn, FADD binds to an initiator caspase (i. e. procaspase 8), resulting in autocatalytic activation that sequentially triggers the execution phase (Chinnaiyan et al., 1995) (Fig. 6). 

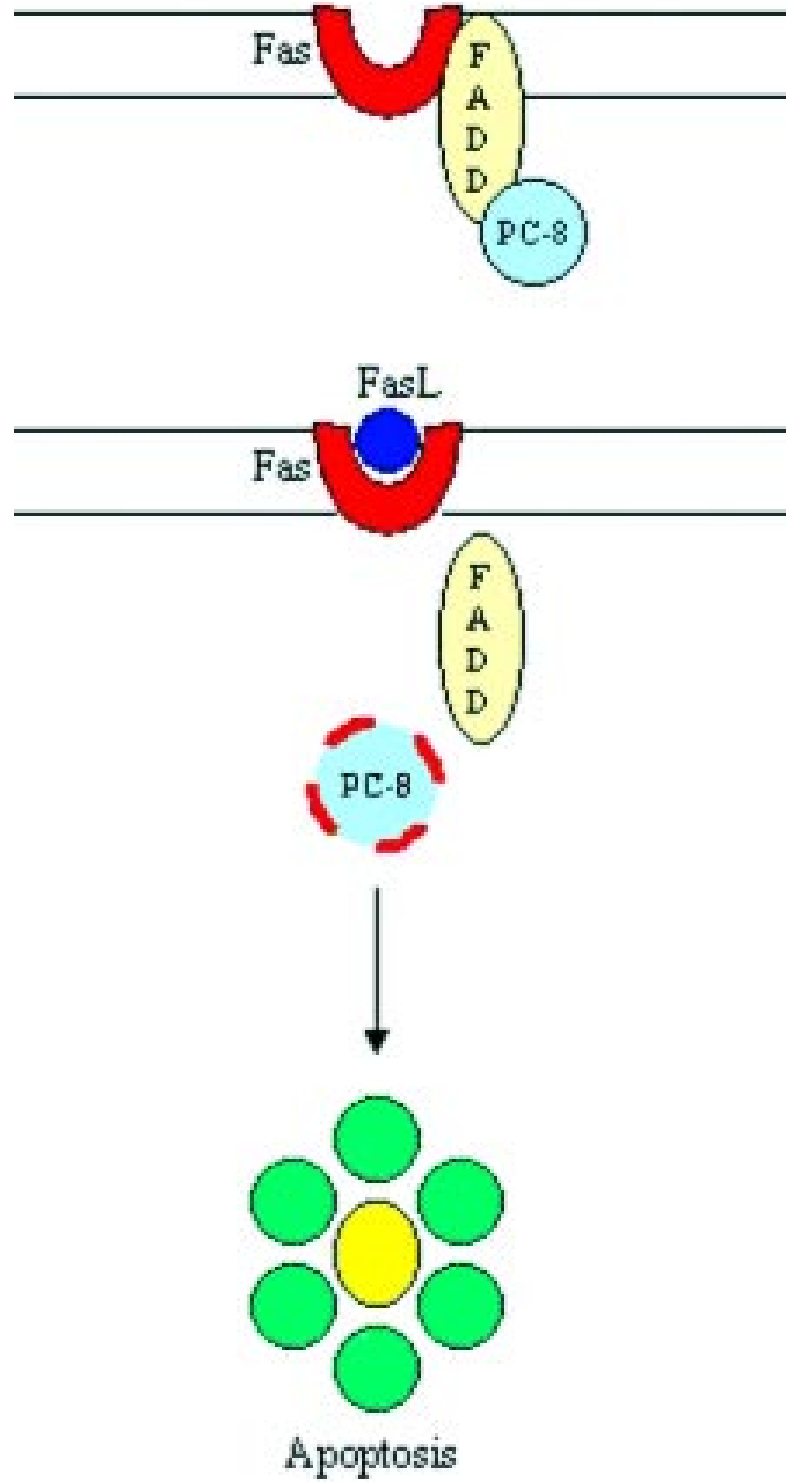

Fig. 6 - Fas is constitutively bound to FADD which in turn binds pro-caspase 8 (PC-8). The interaction between Fas and its ligand determines disruption of Fas-FADD complex and activation of the caspase-cascade.

It has already been demonstrated that Fas/FasL system is one of the most important prognostic factors for the clinical outcome in patients with non-small cell lung carcinomas (Volm and Koomagi, 2000K; Restifo, 2001). Indeed, lung cancers with higher proliferative activity and lower Fas expression determine a worse prognosis compared to tumors with lower proliferative activity and higher Fas expression. Moreover, high FasL levels are associated with an intrinsically worse prognosis of chronic myelogenous leukemia (Ravandi et al., 2001), human breast cancer (Mottolese et al., 2000), gastric cancer (Ohno et al., 2000) and it could be related to tumor progression. In particular, a strong association was shown between FasL expression and metastatic lymph node presence in human breast cancer (Mottolese et al., 2000), while Fas presence correlated to a node-negative status and, therefore, to a longer disease-free survival. In addition, patients with FasL-negative primary lesions tend to exhibit longer survival times than patients with FasL-positive primary gastric carcinoma (Nagashima et al., 2001).

Furthermore, it has been demonstrated that FasL expression in tumor cells may kill the Fas-positive tumor-infiltrating lymphocytes in colorectal carcinoma (Okada et al., 2000), mycosis fungoides (Ni et al., 2001), breast cancer (O’Connell et al., 1999) and pancreatic carcinoma (Ungefroren $\mathrm{H}$ et al., 1999), this representing the potential mechanism of tumor immune escape and progression. In particular, resistance to the Fas/FasL system in breast cancer can be due to the combination of low levels of Fas expression and intracellular defects in death signal transduction. Indeed, Fas sensitivity restoration in breast cancer cell lines after treatment with vitamin E succinate, up-regulating the Fas/FasL system, represented a potential therapy to promote autocrine suicide of tumor cells (Turley et al., 1997). Moreover, Bennett et al. (2001) recently demonstrated that colonocytes acquire resistance to Fas mediated apoptosis early in the adenoma-carcinoma sequence, this being evident in the mild dysplasia. Analogously, chronic ultraviolet radiation exposure may induce a loss of FasL expression in keratinocytes, leading to deregulation of apoptosis and initiating skin cancer (Ouhtit et al., 2000). Although an immune suppression has been shown in T-lymphocytes infiltrating pancreatic cancers, these tumorescape mechanisms could represent a new target for modern treatments (Ungefroren et al., 1999).

In addition, elevated levels of soluble FasL in the serum might be associated with a great risk of disease progression and recurrence in patients with bladder carcinoma (Mizutani et al., 2001) or breast cancer (Ueno et al., 1999). In fact, serum levels of soluble FasL were 2.5-fold higher in patients with invasive bladder carcinoma as compared to patients with superficial tumor (Mituzani et al., 2001). Analogously, a multivariate analysis 
confirmed that the serum level of soluble FasL was an independent prognostic indicator in breast cancer patients (Ueno et al., 1999). In both studies, serum levels of soluble FasL increased with the tumor grade and stage, predicting early recurrence in patients. Finally, the expression of Fas and FasL appeared to have both therapeutic and prognostic implications also in high-grade renal cell carcinoma (Kim et al., 2000) and lung cancer (Koomagi and Volm, 1999); the former because the growth of cancer could be controlled by Fas-mediated apoptosis, the latter because carcinomas that were positive for Fas and FasL showed a greater sensitivity to doxorubicin in vitro.

We studied the expression of Fas in human myelolipoma, a rare benign soft tissue tumor composed of haematopoietic cells, comparing it to normal human bone marrow (Cappello et al., 1999). Fas was strongly expressed both in myelolipoma and normal bone marrow, but apoptosis, revealed in situ with TUNEL reaction, was reduced in myelolipoma compared to bone marrow. These data suggested that the Fas/FasL system in myelolipoma is altered, Fas not being able to trigger the apoptotic machinery. In addition, it provided evidence that the development of benign tumor masses could also be imputable to a defective apoptosis.

Moreover, we recently analysed the pro-apoptotic activity of gemcitabine in three non-small cell lung cancer cell lines, finding an increase of Fas receptor expression in all cell lines after treatment (Pace et al., 2000). Subsequently, we incubated these cells with anti-Fas agonistic monoclonal antibody, inducing cell death and demonstrating that the Fas receptor was active. The possibility to induce the expression of functionally active Fas and to induce apoptosis using immunostimulatory drugs can be a modern therapeutic approach to tumor diseases.

\section{CONCLUSIONS}

A study of 58 different malignant tumors showed that each tumor had a characteristic apoptotic index reflecting innate tumor susceptibility to undergo apoptosis (Staunton et al., 1995). Different elements in the signalling pathways that couple cellular damage to apoptosis can determine this susceptibility, as well as tumoral stadium.

Apoptosis can be economically evaluated in routine H\&E sections or using in situ detection system, i.e. TUNEL; nevertheless, these systems have demon-



Fig. 7 - One of the targets of gene therapy could be the reestablishment of a wild-type p53 in tumor cells, to induce apoptosis and to arrest cancer progression.

strated their failure, and nowadays immunohistochemical staining for pro- and anti-apoptotic markers seems to be more specific. In particular, overexpression of some markers, such as p53, bcl-2, Fas and FasL, showed a prognostic value in a large series of studies. Finally, the combination of more detailed knowledge on carcinogenesis with the possibility to repair lost genes using genetic manipulation techniques seems to be the future tool in cancer therapy.



Fig. 8 - Alternatively to that shown in Fig. 7, the restoration of Fas-induced apoptosis in tumor cells might induce apoptosis, becoming an important tool in tumor therapy. 